\title{
Observation of Self-Induced Optical Vortex Precession
}

\author{
Mohamed El Ketara and Etienne Brasselet* \\ Université Bordeaux, Laboratoire Ondes et Matière d'Aquitaine, UMR 5798, F-33400 Talence, France \\ CNRS, Laboratoire Ondes et Matière d'Aquitaine, UMR 5798, F-33400 Talence, France
}

(Received 6 October 2012; published 6 June 2013)

\begin{abstract}
We report on the observation of self-induced precession of an optical vortex as a result of the nonlinear interaction between light and liquid crystals. The phenomenon corresponds to an instability for the spinorbit interaction of light that manifests as a spontaneous axial symmetry breaking, which leads to the orbital motion of the optical vortex around the beam propagation. A nonlinear spin Hall effect of light is experimentally identified, thereby unveiling an original demonstration of spin to extrinsic orbital light angular momentum self-conversion.
\end{abstract}

DOI: 10.1103/PhysRevLett.110.233603

PACS numbers: 42.50.Wk, 42.50.Tx, 61.30.Gd

A property shared by all waves is the possible existence of phase singularities [1], commonly known as vortices. They are locations in space, points in two dimensions and lines in three dimensions, where the wave amplitude is zero whereas phase takes all values. Since a basic feature of a vortex is the intrinsic circulation of a current, vortex precession represents an intriguing combination of intrinsic and extrinsic degrees of freedom. Precessing vortices can be obtained from appropriate mechanical rotation. Examples can be mentioned in hydrodynamics [2], superfluids [3], matter waves [4] or optical waves [5]. Selfinduced manifestations of the phenomenon may also occur. For instance, in optics, finite rotation of interacting optical vortices has been demonstrated in linear or nonlinear media [6,7] whereas stationary circling of optical vortices has been shown in gain media [8-10]. Here, by using light and liquid crystals, we report on a novel physical mechanism leading to self-induced vortex precession. This involves angular momentum exchanges, on the one hand, between light and matter and, on the other hand, between the spin and orbital degrees of freedom of the light field itself.

More precisely, the optical vortex we observe is initially generated by spin-orbit interaction of a diverging circularly polarized laser field passing through a nematic liquid crystal (NLC) film, similarly to the case of a $c$-cut slab uniaxial solid crystal $[11,12]$. The film is indeed a uniaxial, optically anisotropic nonlinear medium, with homeotropic boundary conditions that preferentially align the director (a unit vector $\mathbf{n}$ that refers to the local averaged molecular orientation) parallel to the beam propagation axis $z$. Light is known to reorient liquid crystals [13], and here it gives rise to a self-induced optical vortex precession phenomenon at high enough intensity, which we identify as a nonlinear spin Hall effect of light. We have characterized the dependence of this phenomenon on the power, transverse cross section, and polarization of the incident light field. The effect is found to be robust and the precession handedness is controlled by the sign of the input spin angular momentum (SAM).

The experimental setup is shown in Fig. 1. A continuous-wave circularly polarized Gaussian light beam with wavelength $\lambda=532 \mathrm{~nm}$, divergence angle inside the medium $\theta_{0}\left(\theta_{0} \lesssim 10^{\circ}\right)$, and incident power $P$ is normally incident onto a $L=57 \mu \mathrm{m}$-thick homeotropic NLC film (E7 material from Merck). The diameter of the beam in the plane of the film, at a distance $z$ from the focal plane, is $d$. The output light is collimated into a beam with radius $w$ and passes through a polarization analyzer, which consists of two electrically controlled birefringent retarders $R_{1,2}$ and a polarizer $P$, before being visualized with a video camera. Appropriate retardances for $R_{1,2}$ give access to the intensity profiles $I_{\beta}(x, y)$ of various polarized components of the output light field, where $\beta=$ $\left(-45^{\circ}, 0^{\circ}, 45^{\circ}, 90^{\circ}\right)$ refers to a linear polarization state oriented at an angle $\beta$ from the $x$ axis and $\beta=(L, R)$ refers to left- and right-handed circular polarization states.

As said above, the NLC film at rest behaves as $c$-cut slab of uniaxial solid crystal; hence, it partially converts the incident SAM into orbital angular momentum (OAM) carried by the contra-circularly polarized output light field component $[11,12]$. This corresponds to the generation of an on-axis optical vortex characterized by an azimuthal

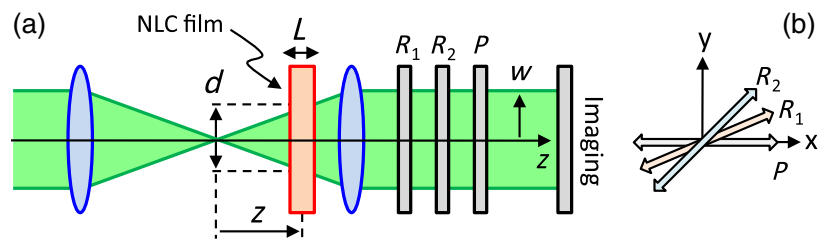

FIG. 1 (color online). Experimental setup. (a) A moderately focused circularly polarized Gaussian beam is normally incident onto a homeotropic NLC film. The output beam is collimated and analyzed using two electrically controlled retarders $R_{1,2}$ and a polarizer $P$. (b) Orientation of the polarizer and of the slow axes of the retarders. 
dependence of the electric field amplitude of the form $\exp (i \ell \phi), \phi$ being the azimuthal angle, where $\ell=2 \sigma$ is called the topological charge and $\sigma= \pm 1$ refers to leftand right-handed circularly polarized incident beam. Since any optical reorientation of the NLC is noninstantaneous, this situation is observed just after the laser beam is turned on, see Fig. 2(a) at $t=0 \mathrm{~s}$, where $I_{R}(x, y)$ is shown for lefthanded incident beam. With present parameters, we measure a $30 \%$ SAM to OAM conversion rate for the NLC at rest, a value close to the $33 \%$ predicted from theory [12].

As the laser beam is turned on, the axially symmetric incident intensity distribution associated with a circular polarization state leads to an axially symmetric torque density distribution exerted on the initial director field that points along the $z$ axis $[14,15]$. The resulting distorted director field is axially symmetric and chiral, whereas the vortex intensity distribution is invariant along $z$, see Fig. 2(a) at $t=10 \mathrm{~s}$. This represents a generalization of the concept of " $q$ plates" [16] — azimuthally patterned optically anisotropic media with uniform birefringent phase retardation - to radially nonuniform birefringent plate, here with $q=1$. In addition, we note that the vortex core has significantly shrunk compared to the undistorted situation while the power of the vortex field has increased and its topological charge is preserved. These observations are the signature of a nonlinear spin-orbit interaction of light associated with self-enhancement of the SAM to OAM conversion [17].

However, above an incident power threshold, axial symmetry spontaneously breaks after a transient and a steady orbital motion of the optical vortex around the $z$ axis is observed. This is illustrated in the bottom panels of Fig. 2(a), where the nonaxisymmetric distorted director field is sketched at $t=110 \mathrm{~s}$. The full vortex dynamics is shown in Fig. 2(b), where the ellipse refers to the steady state trajectory, whose stationarity is illustrated in Fig. 2(c). Noteworthy, the precessing dark spot corresponds to an optical vortex with topological charge $2 \sigma$, as shown for the case $\sigma=+1$ in Fig. 3 where the intensity and phase (namely $\Phi=\arctan \left[\left(I_{+45^{\circ}}-I_{-45^{\circ}}\right) /\left(I_{0^{\circ}}-I_{90^{\circ}}\right)\right]$ following the Stokes polarimetry procedure used in [18]) spatial distribution of the contra-circularly polarized component of the output light field are displayed in panels (a) and (b), respectively. The observed local azimuthal dependence of the phase around the singularity of the form $\Phi=2 \phi$ indeed confirms a topological charge two [see Fig. 3(c)]. This supports a local winding by $2 \pi$ of the projection of the director on the $(x, y)$ plane around an off-axis orientational defect as depicted in Fig. 2(a) at $t=110 \mathrm{~s}$; that is to say a 1-plate with nonuniform birefringent retardation profiles along both the radial and azimuthal coordinates.

Noting that the initial rotational symmetry of the system implies that the $z$ component of the angular momentum of the NLC is a conserved quantity that does not mix with the angular momentum of light [19], the observed self-induced optical vortex precession associated with broken rotational symmetry is related with angular momentum transfer between light and matter. The phenomenon therefore appears as the spin-orbit generalization of the self-induced stimulated light scattering, which originally involved solely the action of SAM of light in a NLC film [20]. The connection with the optomechanical experiment of radial nematic droplets in circularly polarized tweezers, which rotate above a power threshold, should also be mentioned [21].

The threshold character of the self-induced optical vortex precession is demonstrated in Fig. 4(a) that shows the power dependence of $\rho=\langle D\rangle_{t} / w$, where $\langle D\rangle_{t}$ refers to the time-averaged value of the off-axis distance in the observation plane [see Fig. 3(a)]. In this figure, all data points have been obtained by turning on the laser from an initial situation where the NLC is at rest. The abrupt and significant jump for $\rho$ observed at $P=P_{\text {th }}$, namely the offaxis distance increases typically by one order of magnitude with respect to the noise level over $1 \%$ change of the input beam power, unambiguously demonstrates a robust phenomenon. The above threshold behavior indicates that the radius of the orbiting vortex trajectory is roughly constant with input power, see Fig. 4(a). In addition, the power dependence of the precession period $T$ and of the ratio $e$
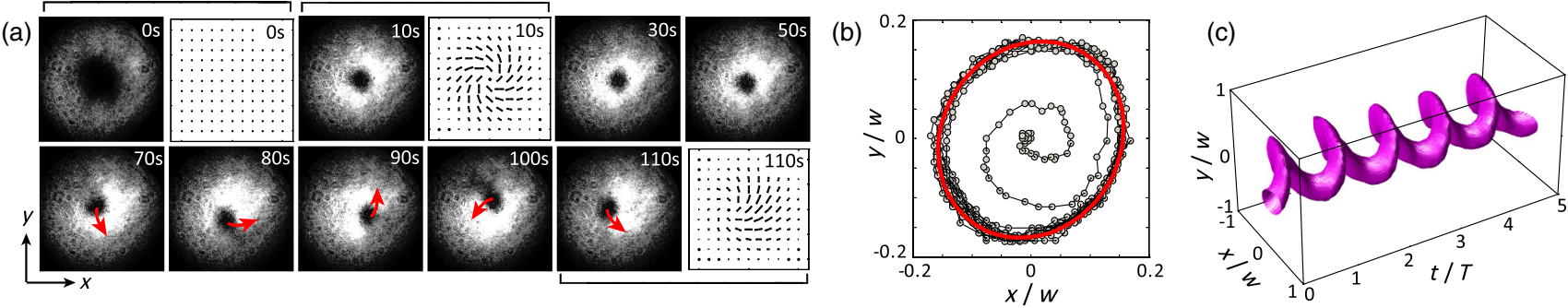

FIG. 2 (color online). Self-induced optical vortex precession for left-handed circularly polarized incident beam. (a) Transient vortex dynamics observed by recording $I_{R}(x, y)$, the input beam being turned on at $t=0 \mathrm{~s}$. Illustrative representations of the projection of the director field on the $(x, y)$ plane, $\|\mathbf{n}-(\mathbf{n} \cdot \mathbf{z}) \mathbf{z}\|$, are shown at $t=0,10$ and $110 \mathrm{~s}$. Arrows on images indicate the motion of the optical vortex. (b) Dynamics of the optical vortex trajectory over $500 \mathrm{~s}$ with time step $d t=1 \mathrm{~s}$. The stationary trajectory is fitted by an ellipse. (c) Steady state three-dimensional trajectory of the precessing optical vortex core, $T$ being the precession period. The experimental conditions are $z=350 \mu \mathrm{m}, \theta_{0}=8.5^{\circ}$ and here $P=780 \mathrm{~mW}$. 


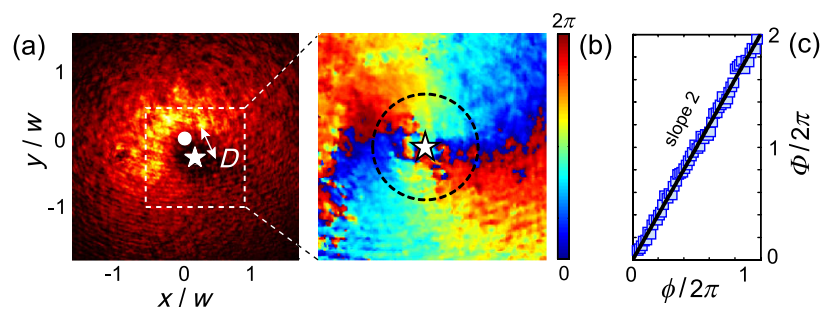

FIG. 3 (color online). (a) Snapshot of the intensity profile of optical vortex field during steady state precession under the experimental conditions of Fig. 2. The circle marker refers to the initial location of the vortex at $t=0$ whereas the star marker refers to the actual location of the optical vortex. (b) Local phase spatial distribution nearby of the optical phase singularity. (c) Azimuthal dependence of the phase along the dashed circle shown in panel (b).

between the minor and the major axes of the elliptical vortex trajectory are shown in Fig. 4(b).

Regarding the ellipticity of the optical vortex trajectory characterized with $e \simeq 0.8$, see Figs. 4(b) and 5(d), it might be argued that it arises from the residual ellipticity of incident Gaussian intensity profile, measured as $e \gtrsim 0.9$. However, we notice that (i) the azimuth of the elliptical trajectory differs for slightly different settings, (ii) there is no clear trend for the power dependence of the azimuth, and (iii) there is no obvious influence of the handedness of the incident circular polarization on $T, \rho$, and $e$, though right- and left-handed trajectories are not identically the same. These observations indicate a sensitivity to residual axial symmetry breaking. The anisotropy of the elastic coefficients might also play a role. It is indeed known to lead to anisotropic reorientation patterns in conventional self-phase-modulation experiments [22]. More recently, broken circular symmetry has also been reported in the context of NLC reorientation under spin-orbit interaction [23]. Further study would be necessary to clarify this issue.

The robustness of the phenomenon is emphasized in Fig. 5, where the dependence of its threshold power, offaxis distance, precession period, and trajectory aspect ratio on the dimensionless aspect ratio $\delta=d / L$ are shown.
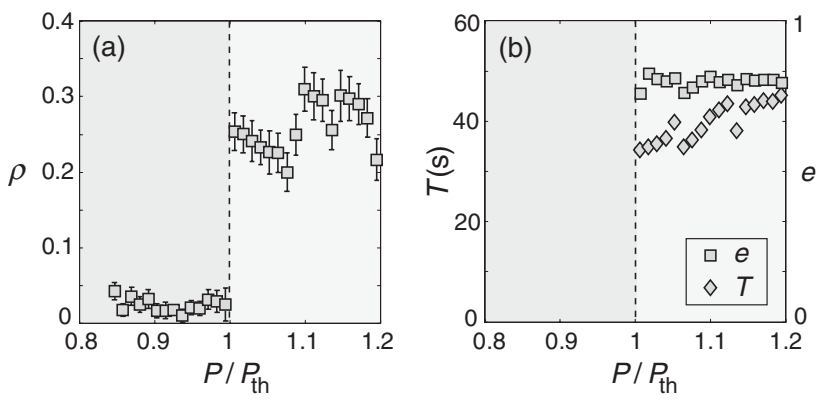

FIG. 4. Power dependence of (a) $\rho$ and (b) $T$ (diamond markers) and $e$ (square markers), see text for definitions. The shadowed area refer to two distinct regimes, below and above the threshold power $P=P_{\text {th }}$. The experimental conditions are those of Fig. 2, for which $P_{\mathrm{th}}=705 \mathrm{~mW}$.
We stress that optical vortex precession always takes place above a well-defined threshold power that increases with the input beam cross section $d, L$ being kept constant [see Fig. 5(a)]. Such a behavior is fairly well described by the standard theory of optical reorientation of NLC films under bounded light beams [24], which predicts an orientational instability threshold power $P_{\mathrm{OFT}}=$ $\pi^{3}(\delta / 2+\sqrt{2} / \pi)^{2} c K n_{\|}^{2} /\left[n_{\perp}\left(n_{\|}^{2}-n_{\perp}^{2}\right)\right]$ [25], where $c$ is the speed of light, $K$ the Frank elastic constant under the assumption of isotropic elasticity, $n_{\|, \perp}$ the refractive index along and perpendicular to the director, and OFT refers to the so-called optical Fréedericksz transition. Taking $n_{\|}=1.756$ and $n_{\perp}=1.528$ at $532 \mathrm{~nm}$ wavelength and $25^{\circ} \mathrm{C}$ temperature [26], the best fit using $K$ as the only adjustable parameter gives $K=12.4 \mathrm{pN}$ [see solid curve in Fig. 5(a)]. The $\simeq 8 \%$ difference with the average value of the experimentally measured splay, twist, and bend Frank elastic constants of $E 7, K=11.4 \mathrm{pN}$ [27], leads us to conclude that the observed self-induced optical vortex precession regime is reminiscent of the homogeneous bifurcation of a NLC film under a plane wave. A quantitative theoretical description of the observations in their entirety is beyond the aim of the present contribution and appears highly challenging in view of the complexity of the involved physical ingredients.

Moreover, although the handedness of the incident circular polarization state has neither an influence on the threshold power value nor on the above threshold behavior, it controls the handedness of the orbiting dynamics of the vortex (not shown here). This implies that the precession phenomenon is driven by optical SAM deposition into the

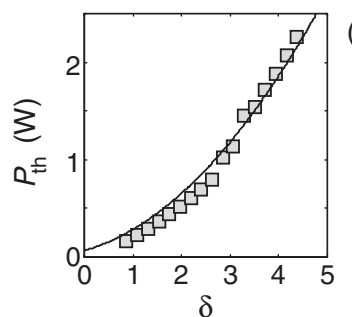

(a)
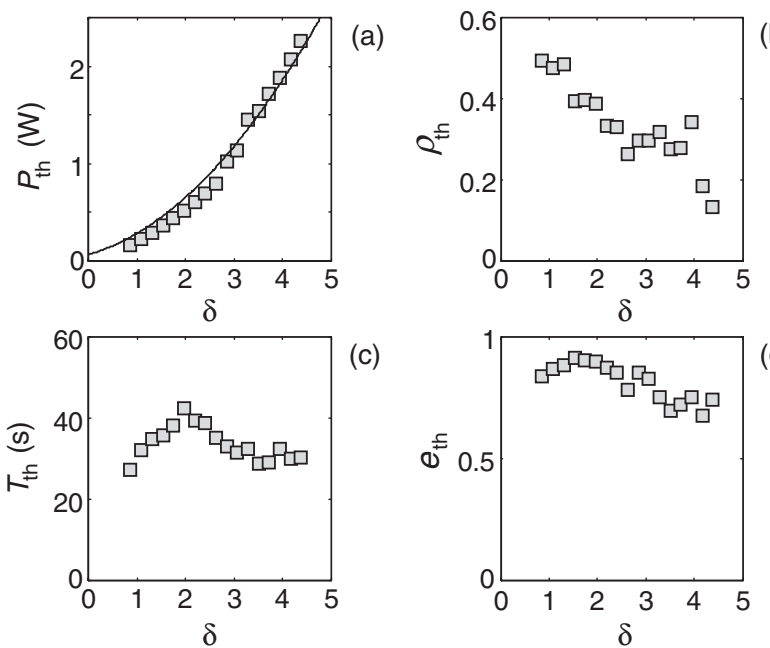

(c)

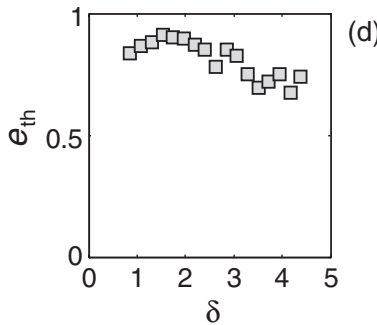

FIG. 5. Optical vortex precession characteristics at the onset of the transition versus $\delta=d / L$. (a) Threshold power $P_{\text {th }}$. (b) Reduced off-axis distance $\rho_{\text {th }}$. (c) Precession period $T_{\text {th }}$. (d) Aspect ratio of the steady state elliptical trajectory $e_{\mathrm{th}}$. The experimental conditions correspond to $\theta_{0}=10.6^{\circ}$. The solid curve in panel (a) is the optical Fréedericksz transition best fit, see text for details. 

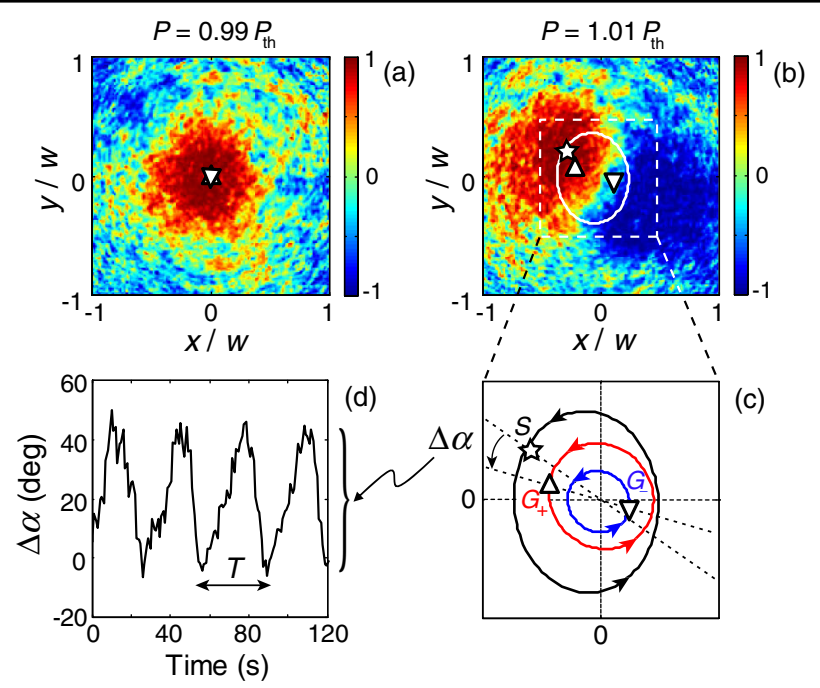

FIG. 6 (color online). Maps of the reduced third Stokes parameter $s_{3}$ for an incident power just below (a) and above (b) the threshold power $P_{\text {th }}$. The ellipse in panel (b) is the steady state vortex trajectory, the star marker being the location of the charge two phase singularity at given time whereas up and down triangle markers refer to the corresponding spin up and down centers of gravity. (c) Enlargement of panel (b) that shows the steady state trajectories of S, $G_{+}$and $G_{-}$. (d) Dynamics of the angle $\Delta \alpha$ over of few precession periods. The experimental conditions correspond to a left-handed circularly polarized incident beam with $\theta_{0}=9.5^{\circ}$ and $z=310 \mu \mathrm{m}$.

liquid crystal. An orbital phenomenon driven by SAM echoes spin Hall effect of light $[28,29]$ - the optical analog of so-called spin Hall effect [30] for electrons. It refers to the polarization dependent trajectory of a light beam, that is to say the interdependence between the SAM and the extrinsic OAM of light. In practice, it usually corresponds to subtle spatial deviations from geometrical optics predictions that can nevertheless be retrieved experimentally with great accuracy [31].

However, the macroscopic (i.e., on the spatial scale of the beam transverse dimension) spatial redistribution of spins-namely, the right- and left-handed circularly polarized components of a light beam-can be readily observed by placing a nanoscatterer slightly off-axis from a tightly focused circularly polarized light beam, as demonstrated in [32]. Noticeably, this has been identified as a giant spin Hall effect of light [32]. In our experiment, the nonlinear uniaxial optically anisotropic medium generate itself a spin-orbit scatterer placed off-axis with respect to beam propagation direction, as sketched in Fig. 2(a) at $t=110 \mathrm{~s}$, from which the manifestation of a nonlinear spin-Hall effect of light is therefore expected. For this purpose, we measure the spatial distribution of the reduced third Stokes parameter $s_{3}=\left(I_{L}-I_{R}\right) /\left(I_{L}+I_{R}\right)$, which exhibits a sudden macroseparation of right- and left-handed circularly polarized components of the output light beam at the onset of the self-induced optical vortex precession transition. This is demonstrated in Fig. 6 where
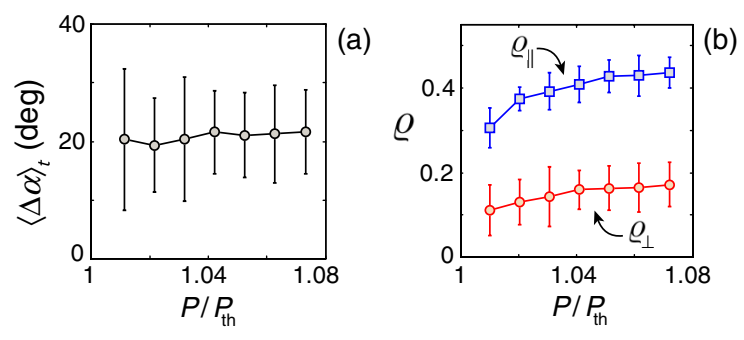

FIG. 7 (color online). (a) Power dependence of the timeaveraged angle $\langle\Delta \alpha\rangle_{t}$ in the steady state optical vortex precession regime. Error bars refer to the standard deviation of the well-defined oscillation of $\Delta \alpha(t)$, as illustrated in Fig. 6(d). (b) Power dependence of $\varrho_{\|, \perp}$. The experimental conditions are those of Fig. 6.

$s_{3}(x, y)$ is shown for an incident power just below $[P=$ $0.99 P_{\text {th }}$, Fig. 6(a) $]$ and above $\left[P=1.01 P_{\text {th }}\right.$, Fig. 6(b) $]$ the threshold power. Indeed the initially superimposed spin up and down centers of gravity $G_{+,-}$, with coordinates $x_{G_{+,-}}=\iint x I_{L, R}(x, y) d x d y / \iint I_{L, R}(x, y) d x d y$ and $y_{G_{+,-}}=$ $\iint y I_{L, R}(x, y) d x d y / \iint I_{L, R}(x, y) d x d y$, are separated by the reduced distance $\varrho=\left\langle\left|G_{-} G_{+}\right|\right\rangle_{t} / w=0.3-0.5$ for $P>P_{\text {th }}$. To our knowledge, this constitutes the first experimental observation of a nonlinear spin Hall effect of light, which represents an original demonstration of selfinduced SAM to extrinsic OAM conversion.

Intriguingly, such nonlinear SAM to extrinsic OAM conversion is associated to a "transverse" effect-a shift of $G_{+,-}$orthogonal to the vortex displacement. This shift is characterized by the angle $\Delta \alpha$ between lines OS and $G_{-} G_{+}$, O being the origin, as depicted in Fig. 6(c). This angle exhibits an oscillating behavior with the same period $T$ as the vortex precession, see Fig. 6(d), with a nonzero the time-averaged value whatever the input beam power above the threshold as shown in Fig. 7(a). This allows for the quantitative evaluation of the latter transverse displacement defined as $\varrho_{\perp}=\left\langle\left|G_{-} G_{+}\right| \sin \Delta \alpha\right\rangle_{t} / w$ whose power dependence is shown in Fig. 7(b), where $\varrho_{\|}=$ $\left\langle\left|G_{-} G_{+}\right| \cos \Delta \alpha\right\rangle_{t} / w$ is also given.

Summarizing, self-induced optical vortex precession has been observed in a nonlinear optics experiment, using a nematic liquid crystal film as the nonlinear medium. The threshold character of the phenomenon has been unambiguously demonstrated, the input beam power being the control parameter. Self-induced vortex precession has been shown to be associated with a nonlinear spin Hall effect of light, thereby unveiling an original manifestation of spin to extrinsic orbital light angular momentum self-conversion.

We thank K. Bliokh for fruitful discussion.

*e.brasselet@loma.u-bordeaux1.fr

[1] J. F. Nye and M. V. Berry, Proc. R. Soc. A 336, 165 (1974).

[2] O. Lucca-Negro and T. O'Doherty, Prog. Energy Combust. Sci. 27, 431 (2001). 
[3] R. J. Zieve, Y. Mukharsky, J. D. Close, J. C. Davis, and R. E. Packard, Phys. Rev. Lett. 68, 1327 (1992).

[4] M. R. Matthews, B. P. Anderson, P. C. Haljan, D. S. Hall, C. E. Wieman, and E. A. Cornell, Phys. Rev. Lett. 83, 2498 (1999).

[5] T. A. Fadeyeva, A. F. Rubass, B. V. Sokolenko, and A. V. Volyar, J. Opt. A: Pure Appl. Opt. 11, 094008 (2009).

[6] B. Luther-Davies, R. Powles, and V. Tikhonenko, Opt. Lett. 19, 1816 (1994).

[7] D. Rozas, C. T. Law, and J. G. A. Swartzlander, J. Opt. Soc. Am. B 14, 3054 (1997).

[8] A. B. Coates, C. O. Weiss, C. Green, E. J. D’Angelo, J. R. Tredicce, M. Brambilla, M. Cattaneo, L. A. Lugiato, R. Pirovano, F. Prati, A. J. Kent, and G.-L. Oppo, Phys. Rev. A 49, 1452 (1994).

[9] M. Vaupel and C. O. Weiss, Phys. Rev. A 51, 4078 (1995).

[10] F. Prati, L. Zucchetti, and G. Molteni, Phys. Rev. A 51, 4093 (1995).

[11] A. Volyar and T. Fadeyeva, Opt. Spectrosc. 94, 235 (2003).

[12] A. Ciattoni, G. Cincotti, and C. Palma, J. Opt. Soc. Am. A 20, 163 (2003).

[13] N. V. Tabiryan, A. V. Sukhov, and B. Y. Zel'dovich, Mol. Cryst. Liq. Cryst. 136, 1 (1986).

[14] E. Brasselet, Opt. Lett. 34, 3229 (2009).

[15] E. Brasselet, J. Opt. 12, 124005 (2010).

[16] L. Marrucci, C. Manzo, and D. Paparo, Phys. Rev. Lett. 96, 163905 (2006).

[17] M. E. Ketara and E. Brasselet, Opt. Lett. 37, 602 (2012).
[18] E. Brasselet, N. Murazawa, H. Misawa, and S. Juodkazis, Phys. Rev. Lett. 103, 103903 (2009).

[19] A. Ciattoni, G. Cincotti, and C. Palma, Phys. Rev. E 67, 036618 (2003).

[20] E. Santamato, B. Daino, M. Romagnoli, M. Settembre, and Y. R. Shen, Phys. Rev. Lett. 57, 2423 (1986).

[21] N. Murazawa, S. Juodkazis, and H. Misawa, J. Phys. D 38, 2923 (2005).

[22] A. S. Zolot'ko, V. F. Kitaeva, N. Kroo, N. N. Sobolev, and L. Chillag, JETP Lett. 32, 158 (1980).

[23] E. Brasselet, Phys. Rev. A 82, 063836 (2010).

[24] A.S. Zolot'ko, V.F. Kitaeva, V. A. Kuyumchyan, N. N. Sobolev, and A.P. Sukhorukov, JETP Lett. 36, 80 (1982).

[25] Present expression is twice the one of Ref. [24], accounting for the fact that the polarization state is circular.

[26] J. Li, C.-H. Wen, S. Gauza, R. Lu, and S.-T. Wu, J. Disp. Technol. 1, 51 (2005).

[27] J. F. Stromer, E. P. Raynes, and C. V. Brown, Appl. Phys. Lett. 88, 051915 (2006).

[28] M. Onoda, S. Murakami, and N. Nagaosa, Phys. Rev. Lett. 93, 083901 (2004).

[29] K. Bliokh and Y. Bliokh, Phys. Lett. A 333, 181 (2004).

[30] J. E. Hirsch, Phys. Rev. Lett. 83, 1834 (1999).

[31] O. Hosten and P. Kwiat, Science 319, 787 (2008).

[32] O. G. Rodriguez-Herrera, D. Lara, K. Y. Bliokh, E. A. Ostrovskaya, and C. Dainty, Phys. Rev. Lett. 104, 253601 (2010). 